\title{
A Survey of Multipath Routing Schemes of Wireless Mesh Networks
}

\author{
S.K. Gurung \\ Department of Computer Science \\ Saint Mary's College \\ Shillong, Meghalaya , India
}

\author{
D.K. Saikia \\ Department of Computer Science and Engineering \\ Tezpur university \\ Napaam, Assam, India
}

\begin{abstract}
There have been many advances in the area of routing in wireless mesh networks and a multitude of routing algorithms have been proposed. In the recent past, a class of routing protocols called multipath routing has gained favour amongst researchers. As opposed to uni path routing where one single path is used to send data from a source to a destination, in multipath routing, multiple paths are used to route data. The idea of using multiple paths to deliver data lends itself well to a wireless multihop network, given its broadcast nature and high connectivity and can offer significant advantages over traditional single path routing - increased reliability, ability to load balance data flows which improves network performance, allows quick recovery from route failures and throughput aggregation. This paper provides brief overviews of the protocols that employ this method and identifies the challenges involved in such a routing strategy.
\end{abstract}

\section{General Terms}

Multi-path routing, Wireless Mesh Networks.

\section{Keywords}

Wireless mesh networks, multipath routing, multihop networks, routing protocols, alternative routes

\section{INTRODUCTION}

Wireless Mesh Networks (WMNs) have assumed significance in recent years as a low cost and fast deploying wireless connectivity solution. Their ease of deployment and self configuring, self-healing, highly reliable and fault tolerant nature makes them suitable for applications such as broadband home networking and community and enterprise wireless networks. WMNs comprise wireless mesh routers and clients and are classified into three broad categories- Infrastructure, Client, and Hybrid [2]. Despite their advantages WMNs face many challenges. They suffer from bandwidth limitations due to interference on the wireless links which results in suboptimal network performance. WMNs are also afflicted with link failure and faulty nodes more frequently than wired networks. Single path routing solutions follow the "best path to a destination" paradigm. However, this may result in the best path being overloaded over time leaving other nodes underutilised. An approach that balances the overall network load is desirable. In recent years a class of routing protocols has emerged that aim to better utilise network resources by exploiting multiple paths that may exist between source and destination. Such protocols distribute data amongst these paths which load balances the traffic, provides fault tolerance, improves error resilience and make better use of network resources. In this paper a review of multipath protocols for wireless mesh networks is presented.

\section{MOTIVATION}

Survey of multipath routing protocols for mobile ad hoc networks are presented in $[23,1,22,15]$ but an examination of multipath routing in WMNs is lacking in the literature. Consequently, a representative set of multipath routing protocols for WMNs is reviewed

\section{MULTIPATH ROUTING}

Multipath routing deals with the problem of selecting diverse paths between source and destination pairs and distributing traffic amongst these routes. In MANETs, multipath routing was seen to increase aggregate bandwidth, provide better error resilience, low latency in recovering from route failures and load balancing [23]. Given the inherent similarities between MANETs and WMNs, it was only natural that multipath routing was adapted to the WMN scenario. WMNs are envisioned to be used in application scenarios such as multimedia data transmission and VOIP applications and multipath routing can provide significant performance improvements over traditional single path routing. The next section elaborates on the advantages of multipath routing.

\subsection{Advantages}

3.1.1 Reliability.

Multipath routing can offer a high degree of reliability by sending redundant data over alternate paths. In case of link failure on one of the paths data may still reach the destination via alternate routes. Schemes that discover multiple paths that are spatially disjoint are more resilient in situations where nodes in a certain area fail at the same time.

\subsubsection{Load Balancing.}

Multiple paths balance the load by carefully distributing traffic over them. Such a strategy, increases overall network throughput by avoiding congested areas.

\subsubsection{Low Latency.}

In uni-path on-demand routing, detection of path breakage leads to costly route discovery procedure and data transmission is halted until a new route is found. In the multipath scenario nodes simply switch to a backup route eliminating the latency in route discovery.

\subsubsection{Throughput Aggregation.}

The data flows over multiple paths can be aggregated to provide services for bandwidth intensive applications such as multimedia transmissions or VOIP communication. When a single path cannot guarantee the bandwidth requirements of a particular application, use of multiple paths to route data can be a way to allow such applications to run in WMNs.

\subsection{Components}

There are three main elements in multipath routing, viz. Path Discovery, Traffic Distribution and Path Maintenance. 


\subsubsection{Path Discovery.}

The path discovery component is responsible for the selection of multiple paths between source-destination pairs. Multiple Paths are distinguished based on the property of disjointedness. Node-disjoint paths have no intermediate nodes in common and are resilient to node outages whereby failure of certain nodes only breaks the paths that flow through those nodes. Link disjoint paths have no links in common but may share nodes. They are not as reliable as node-disjoint ones as failure of a node disrupts all links incident on the node. Node-disjoint routes may not always be available, especially in low node density WMNs. To overcome this problem, Lee et al introduced the idea of using maximally node disjoint paths in [14] where the number of links they have in common is at a minimum.

\subsubsection{Traffic Distribution}

There are three constituent parts of the traffic distribution component. They aim to answer three fundamental questions respectively - How are the paths used? How is the traffic distributed between the paths? and What is the allocation granularity?

Path usage determines whether all the discovered paths or a subset are used and if they are used concurrently [3, 8, 10, 5] or based on some other scheduling scheme such as the use of the alternate path as a backup of the primary path [4].

Traffic distribution is concerned with the quantum of data that is apportioned to multiple paths. The aim is to balance the load between the available routes. A uniform distribution strategy such as a round robin scheme equally distributes the available traffic [17]. Whereas a non-uniform traffic distribution scheme decides the amount of traffic allotted to each path as a function of certain metrics such as the congestion level of nodes along the path $[16,13]$.

Allocation granularity, decides whether the traffic is to be distributed on a per packet [9] or per connection basis. Per connection means that all packets of a connection are sent along the same chosen path. Per packet allocation strategy on the other hand, may choose different paths for different packets. Krishnan et al have shown that per packet allocation allows finer control of network resources and therefore provides better performance [12].

\subsubsection{Path Maintenance.}

Wireless links are unreliable and paths found during the discovery phase may cease to exist due to node or link failures. Path maintenance deals with the discovery of alternate paths when existing paths fail. Path discovery procedures may be initiated either when one path fails or it may be delayed till all paths fail. The latter strategy adds latency and may be unsuitable for QoS sensitive applications, while the former entails performing path discovery every time a single route fails which incurs high control overheads. A suitable trade-off must be chosen by the routing protocol.

\subsection{Issues in Multipath Routing}

While there are many benefits of multipath routing, challenges still have to be addressed. It has been argued that unless a large number of paths are discovered load-balancing in multipath routing may not be achieved [6]. However, it has also been shown that such conclusions may be valid only for scenarios where nodes continuously send data to other nodes via shortest paths [8] and traffic patterns in WMNs do not belong to this model as data flows primarily from nodes to gateways.

Traditional TCP does not perform well when multiple paths are used to route data $[7,11]$. Packets of a flow routed through multiple paths may arrive out of sequence at the destination, leading TCP to assume congestion and trigger congestion control by reducing the data transmission rate. Another problem is that when multiple paths are used each path will have a different round trip time (RTT) which leads to inaccuracies in TCPs RTT estimation. To overcome these problems some researchers have suggested the use of multipath aware TCP such as SCTP [18] and have contended that such TCP coupled with multipath routing leads to significant performance benefits.

Another serious problem afflicting multipath strategies is that of route coupling. It occurs when multiple paths of a flow lie close to each other, typically along the shortest path, resulting in inter-path interference. To overcome the negative effects of route-coupling, solutions such as using multiple channels to build contention-free paths [19] and use of directional antenna [20] have been proposed. However such solutions require additional resources which may not be feasible. Schemes such as [8] aim to tackle the route-coupling problem by using a two round route discovery process to establish a protective region of "in-region nodes" around the primary path. Others use interfering links to provide a protection path around the primary path [10]. Still others make sure that paths are sufficiently spaced apart in terms of spatial distance to ensure that they are inter-path interference free [5].

\section{OVERVIEW OF MULTIPATH ROUTING ALGORITHMS \\ 4.1 MMESH}

Nandiraju et al present a multipath hybrid routing protocol coupled with a traffic splitting algorithm that load balances the traffic [17]. MRs on receiving gateway advertisement messages set up paths to these gateways in order of their performance based on a metric (such as ETT, load etc. . .) MRs then send a PARENT NOTIFICATION message containing information of all chosen routes, to their parent MRs from whom they have received the advertisements. In this manner child MRs notify their parent MRs which paths to use for forwarding traffic and also enables parent MRs to establish reverse routes to the child. CHILD NOTIFICATION messages is then sent by the parent MR to all MRs that are part of the selected routes which is propagated all the way to the gateways. This notification informs all intermediate MRs of child MR and the path to reach the child MR. In this manner multiple routes from MRs to gateways are constructed. Route maintenance is done by MRs periodically monitoring the paths that pass through it and immediately informing its neighbours if it encounters new or stale routes. On detection of a failed next hop an MR suspends the transfer of data through the node for a threshold time pending the recovery of the node when the route is made active again. If the node does not recover within the threshold time the source is notified via a route error message.

MMESH offers two methods for traffic distribution - the first is the round robin scheduling scheme that routes every packet to a different next hop. The second method adds a congestion aware component that looks at the average queue length of the next hop node and based on the load sends the packet or temporarily skips that node and uses an alternate route. However, the protocol does not address the route coupling 
problem. It is designed for a single radio mesh network and therefore does not exploit the benefits of multi-radio architecture. Furthermore it does not support splitting of data of a single flow to multiple gateways.

The authors analyse the working of the protocol through simulations. They show that in comparison to the ADOV protocol, throughput of flow increase significantly when congestion aware MMESH is used.

\subsection{ASMRP}

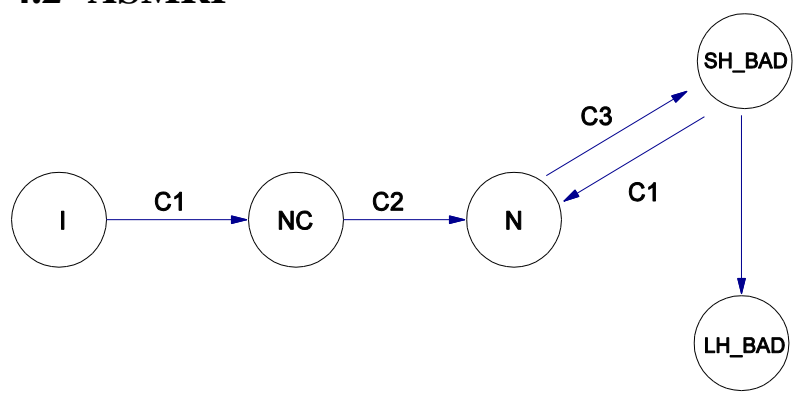

Fig. 1. ASMRP Neighbour state machine [16]

Nandiraju et al extend MMESH to a multi-radio mesh architecture and present an Adaptive State-based Multi-radio Multi-channel Multipath Routing Protocol (ASMRP) [16]. The mesh network is modelled as a multi-level hierarchical structure with the gateways at level 0 . MRs below the gateway are assigned levels based on the hop distance from the gateway. Each MR is equipped with four radios - One radio is used for connecting the router with its client nodes. Another is used to send and receive broadcast messages and the remaining two radios are used for sending and receiving data. The receiving radio is tuned to a fixed channel while the sending radio is switchable and keeps changing channels. To communicate, a MR tunes its sending radio to the channel on which the receiver radio of the peer router is operating by consulting a Neighbour Channel Table (NCT) that is maintained in each MR. The NCT records the channel frequency neighbouring MRs receiver radio are tuned to. Route setup and traffic distribution are based on MMESH. Additionally, ASMRP uses a state machine to maintain multiple routes. MRs can exist in any of the states depicted in figure 1 and transitions occur when certain conditions are satisfied. MRs transition from one state to another when certain conditions are satisfied. A node is in the initial state "I" when it boots. On receipt of any HELLO message (condition C1), a MR moves to the neighbour candidate (NC) state and it can be used as a potential next hop. An MR is in the neighbour $(\mathrm{N})$ state when the link between it and the current MR is stable. MRs transit to this state when a number of HELLO messages are received from it (condition C2). A neighbour of an MR enters the Short-term history bad ( $\mathrm{SH}$ BAD) state when its link with the MR is not stable for short period of time (condition C3). When a neighbour enters this state all routes through it is temporarily disabled till the link improves again and the node moves back to $\mathrm{N}$ state. However if an MR has no other route it will still use the SH BAD neighbour to route data. LH BAD is the state of a neighbour when the link from the MR to it is bad over a longer period of time (condition $\mathrm{C} 4$ ). In such an event all routes through the affected neighbour is deleted from the neighbour list and the MR looks to form alternate routes. By designating a state to each MR, ASMRP determines which nodes may be used to route data. Another optimisation ASMRP proposes is to store routes and additional state information in the intermediate
MRs assigning labels to the routes and sending out these labels in periodic announcement, avoiding the large network overhead of source routing protocols.

ASMRP provides improvement over MMESH due of the use of multiple radios which mitigates the problem of route coupling. However the extent of the improvement depends on the channel assignment scheme employed. By authorising intermediate nodes to route data over to alternate routes when a node or link failure is detected, ASMRP provides local recovery from route failures which improves its performance vis-sa-vis similar protocols. A big disadvantage however, is that it does not support multiple gateways. NS-2 simulator is used to compare the performance of ASMRP with AODV, MMESH, MMR, and CAM-ASMRP. ASMRP provides better aggregate network throughput than the other protocols. It also outperforms other protocols with regards to packet delay due to the use of multiple radios that can provide full duplex transmissions. By using congestion aware traffic distribution strategy, ASMRP provides better packet loss ratios and increases reliability of data delivery.

\subsubsection{CPR}

A concurrent path routing algorithm $-2 \mathrm{CPR}$, is proposed in [3] that seeks to minimise the route coupling problem. It seeks to construct two routing paths from a source to two gateways such that packets can be transmitted concurrently with minimum inter-path interference. Figure 2 shows two paths from source $S$ to gateways 1 and 2 . Nodes $b_{1}$ and $b_{2}$ are the first nodes in the two paths that are outside the interference range of $\mathrm{S}$ and are known as boundary nodes. Nodes $c_{1}$ and $c_{2}$ known as a critical pair, are nodes in the two paths such that the transmissions in the sub-paths $c_{1} \rightarrow b_{1}$ and $c_{2} \rightarrow b_{2}$ are interference free from each other. Consequently paths from nodes to two gateways in a mesh network can be viewed as a concatenation of three sub paths.

$$
p_{i}=S \rightarrow c_{i} \oplus c_{i} \rightarrow b_{i} \oplus b_{i} \rightarrow G_{i}
$$

As sub paths $S \rightarrow c_{1}$ and $S \rightarrow c_{2}$ interfere with each other and

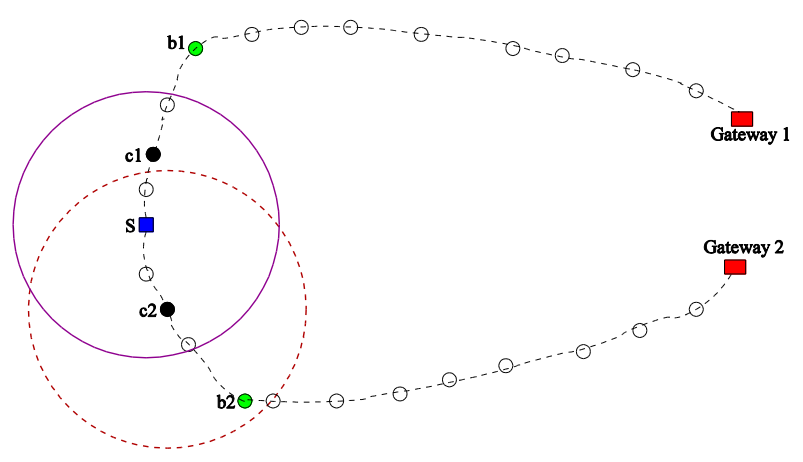

Fig 2. 2CPR from Source $S$ to Gateways 1 and 2. Circle and dotted circle represent interference ranges of source $S$ and node $\mathrm{c} 2$ [3]

cannot be activated simultaneously, packets will take a total time $t\left(S \rightarrow c_{1}\right)+t\left(S \rightarrow c_{2}\right)$ to reach the critical nodes from the source. Sub paths $c_{1} \rightarrow b_{1}$ and $c_{2} \rightarrow b_{2}$ can be activated simultaneously since they are outside the interference range of each other and therefore packets travelling along these sub paths will take time max $\left\{t\left(c_{1} \rightarrow b_{1}\right),\left(c_{2} \rightarrow b_{2}\right)\right\}$ to reach the boundary nodes. Therefore the total expected forwarding times for two packets to travel from source to the boundary nodes in two paths can be expressed as $\mathrm{X}$ 


$$
\operatorname{InnerEFT}(E)=\sum_{p_{i} \in E} t\left(S \rightarrow C_{i}\right)+\max _{p_{i} \in E}\left\{t\left(c_{i} \rightarrow b_{i}\right)\right\}
$$

where, $E=\left\{p_{i}: i=1,2\right\}$. The time taken by the packets to reach the gateways from the boundary nodes is determined by the sub path that has the maximum end-to-end delay and is expressed by

$$
\operatorname{OuterEFT}(E)=\max _{p_{i} \in E}\left\{t\left(b_{i} \rightarrow G_{i}\right)\right\}
$$

Consequently the metric for selecting two concurrent paths can be expressed as

$$
2 C P M(E)=(1-\alpha) \cdot \operatorname{InnerEFT}(E)+\alpha \cdot \text { OuterEFT }(E)
$$

The protocol proposes a solution for the route coupling problem but does not allow for local recovery in case of route failures. Comparisons through simulations with 2SPR and AODV-DM show that 2CPR increases the probability of finding routing paths for a source $\mathrm{S}$ and when the network has high node density, 2CPR improves network throughput by a significant amount.

\subsection{AODV-DM}

$\mathrm{Hu} \&$ Lee present an AODV based multipath routing protocol that constructs node-disjoint multiple paths in [8]. Route coupling is avoided by constructing an insulating region around the primary path by following a two round routediscovery process. To discover the primary path a route request (RREQ) packet is flooded in the network. Intermediate nodes on receiving the RREQ record the information in their routing table and re-broadcast only the first received RREQ. The destination may receive multiple RREQ from different paths but will only respond to the RREQ that arrived on the shortest path via a primary route reply (pRREP) packet. This shortest path is now designated as the primary path. The pRREP is propagated back to the source by following the shortest path via which it had arrived. Intermediate nodes broadcast the pRREP to their neighbour nodes who mark themselves as "in-region" nodes. When the pRREP reaches the source a primary path is established together with the "in-region" nodes that form an insulating region around the primary path. Nodes in the in-region then broadcast region protection (RPRT) packets.

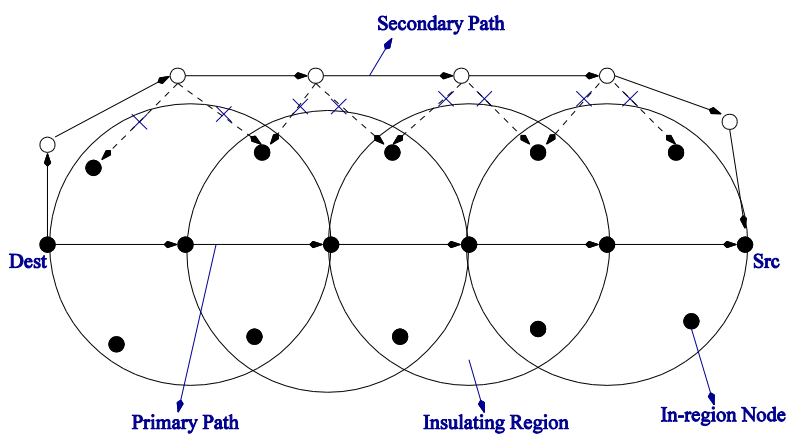

Fig. 3. Separation of primary and secondary paths in AODV-DM. [8]

Neighbours outside the insulating region that receive the RPRT packets remove the in-region nodes from their routing table to prevent future RREPs from entering this region. Figure 3 shows an illustration of AODV-DM depicting the separation of the primary and secondary paths by the insulating region. After the establishment of the primary path the destination responds to other RREQs by sending a secondary route reply (sRREP). sRREPs are propagated back in a manner similar to that of pRREP, the exception being that a node receiving the sRREP does not mark itself as an "inregion" node. On receipt of the sRREP intermediate nodes broadcast the packet and are removed from their neighbours routing tables. This is shown via dashed lines in figure 3 . When a node receives a sRREP but cannot find a node in its RREQ table to forward the packet to it sends a route reply rejection (RREJ) packet to the sRREP sender which then tries other entries in its RREQ table. The authors propose a path aware SCTP scheme to partner the AODV-DM protocol. Specifically the authors recommend path-based congestion control techniques be used to support concurrent use of multiple paths.

The protocol addresses the route coupling problem but the two round route discovery method adds high latency. The use of the insulating region removes a number of nodes from being able to forward data which makes it unsuitable for sparse networks. Another disadvantage of the protocol is that it does not recover from link failures locally. In case a node fails a route reject (RREJ) packet is sent back to the sender to ask it to try other routes. Using OPNET, the authors compare their protocol with AODV and AODVM under different scenarios. With UDP traffic under different transmission rates it was shown that AODV-DM provides better success rates and end to end packet delays. With path aware SCTP, the congestion window oscillation is seen to be reduced under AODV-DM than with AODVM. AODV-DM is also seen to provide better goodput than AODV.

\subsection{MHRP}

MHRP [21] is a hybrid routing protocol for WMNs that incorporates both reactive and proactive elements. It comprises four components.

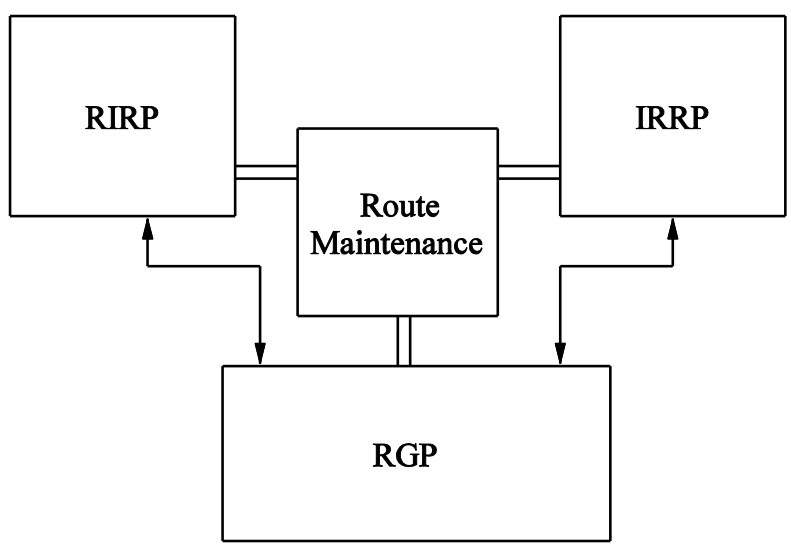

Fig. 4. MHRP Architecture [21]

Intra Region Routing Protocol (IRRP): IRRP, a multipath variation of AODV functions within the ad hoc client mesh network of a hybrid WMN. An Intermediate node receives RREQs from a source via multiple paths. It processes the RREQ packets to create multiple reverse paths to the source and forwards the RREQs. When sending a RREP a destination creates multiple disjoint routes to the source. When a route fails an alternate route is computed at the intermediate node decreasing route recovery latency.

Router Infrastructure Routing Protocol (RIRP): The proactive RIRP is used for routing packets in the relatively static backbone section of WMN. It periodically sends HELLO packets to keep up-to-date routes in the mesh routers. Each node maintains multiple paths to all other nodes in the backbone and selects the best path for routing data. Link 
failures result in a node choosing an alternate route from its routing table.

Region Gateway Protocol (RGP): When nodes in two ad hoc regions need to communicate, RGP is used. RGP creates multiple paths between them by getting routing information from RIRP and IRRP as shown in figure 5. When RGP receives a route request message from a node in the source ad hoc region (message 1) it uses RIRP and IRRP to get route information of the mesh backbone and destination ad hoc region respectively (message 2,3 and message 4,5). With the acquired information RGP constructs multiple routes and sends them to source (message 6).

Route Maintenance Protocol is responsible for the maintenance of routes and for providing alternate routes whenever required.

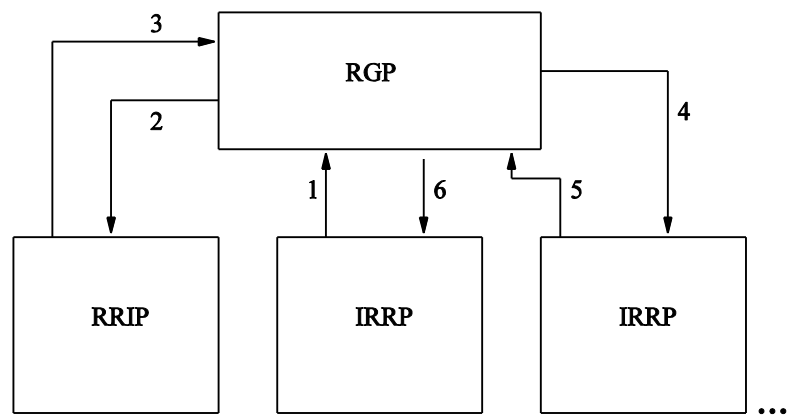

Fig. 5. Flow of route message within MHRP routing components [21]

MHRP routes data by following a three stage procedure. In the first stage IRRP is used to check if the route request by a source can be replied to by a node in the ad hoc region. If not the request is forwarded to RGP running on the router connected to the ad hoc region. If the destination is in another ad hoc region, RGP uses RIRP to determine if a route exists to the destination ad hoc region. If so, RGP queries the IRRP of the destination ad hoc region to find routes to the node. In the second stage, the complete routes from source to destination are constructed with the acquired information. Lastly in the final stage, a route reply containing the multiple paths is sent back to the source.

MHRP allows nodes to select alternative routes from their routing table when link failures are detected both in the ad hoc region and the backbone mesh. This affords faster recovery from route failures. However it does not address the route coupling problem. Simulations show that MHRP provides better throughout than other contemporary routing protocols. MHRP eliminates the need for periodic authentication and consequently decreases routing overhead.

\subsection{DIPRO}

Interference is one of the chief causes of sub-optimal performance of WMNs. Recent studies have concentrated on methods to avoid interference. But contrary to the traditional approach, DIPRO [10] utilises interfering links in the vicinity of the primary path to provide a protection path for it. This is based on the premise that the primary and protection paths are never used at the same time. The protection path acts as a separator between primary paths thereby reducing interference and improving performance. To decrease the consumption of links as protection paths, DIPRO re-uses one protection link to protect many primary paths. To find a primary path for a request, each edge in the network is assigned an edge weight

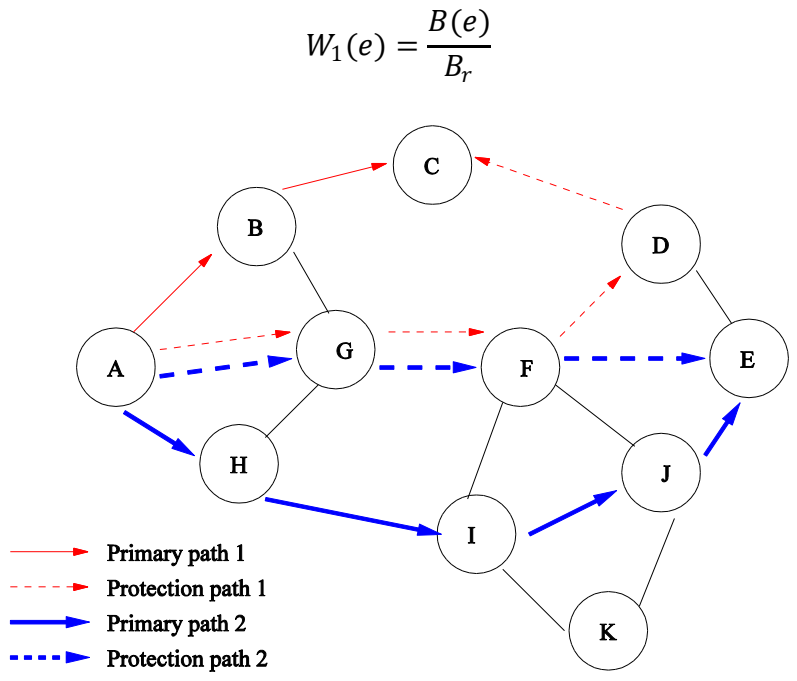

Fig. 6. Primary and Protection Path of DIPRO [10]

where $B(e)$, is the residual bandwidth and $B_{r}$ is the requested bandwidth. Then the shortest path algorithm is used to determine the primary path. If a primary path cannot be found the request is dropped. Once a primary path is determined, a protection path is found for it. The protection path uses the interfered edges of the primary path. First all links that are part of any primary path are removed from the computation of a protection path. Then for each remaining edge $\mathrm{e}$, an interference degree $I(e)$, is calculated, which is the number of edges on the primary path that interferes with e together with a re-usability value $U(e)$, which indicates how many times it has been used as a protection link. Based on these values weight

$$
W_{2}=\alpha \cdot \frac{B_{r}}{B(e)}+\beta \cdot \frac{1}{I(e)+1}+\gamma \cdot \frac{1}{U(e)+1}
$$

is assigned to each edge $e$. Apart from the interference degree and reusability value the weight also includes the bandwidth ratio. The influence of the three components on the overall metric is controlled by giving each factor a weight $\alpha, \beta, \gamma$ respectively. Using the assigned weights a shortest path algorithm is used to find the protection path. If no such protection path is found the request is dropped.

Figure 6 shows two primary and protection paths discovered by DIPRO. Primary paths $A \rightarrow B \rightarrow C$ and $A \rightarrow H \rightarrow I \rightarrow$ $J \rightarrow E$ are separated by protection paths $A \rightarrow G \rightarrow F \rightarrow$ $D \rightarrow C$ and $A \rightarrow G \rightarrow F \rightarrow E$. Links $A \rightarrow G$ and $G \rightarrow F$ are reused by the protection paths. By reusing links, DIPRO improves over AODVDM in network resource utilisation. Also by separating the primary paths using a protection path, DIPRO minimises the route coupling problem. However, in case of failure of the primary path, protection paths are used as a backup route and in such situations inter-path interference may occur.

DIPRO uses two metrics to evaluate the scheme- satisfied ratio and running time. A request is satisfied when link disjoint paths can be found for it. Satisfied ratio is the measure of the number of satisfied requests to the total number of dynamic requests. Running time is the time taken to satisfy a request. Through simulations under different scenarios of varying node density, the authors show that DIPRO performs better than DPR and AODV with regards to the two above metrics. 


\subsection{PATR}

Path-Alternation based Throughput Regulator (PATR) [4], uses multipath routing to increase the reliability of TCP connections in WMNs. Two paths- primary and alternate are constructed and PATR switches to the alternate path, when it encounters congestion in the primary path. Traffic is then routed through the alternate path which avoids congested areas and ensures robust connections. PATR enhances RMAODV, the on demand routing component of Hybrid Wireless Mesh Protocol (HWMP), used in 802.11s mesh networks, to support multipath routing. The enhanced protocol called MRMAODV combines the approaches of AODVM [24] and AODV-DM [8] to create node disjoint paths. Like AODVM a route request (RREQ) table stores the incoming RREQs. Extra fields such as "alm" and "one-hop-alm" are added to the table to enable layer 2 radio aware routing. The route reply (RREP) packet is also appended with additional fields - "reroutemark", "inregion-count", "upstream-node" and "primarypaths-flag". These fields assist in the discovery of multiple paths, and in the separation of the primary path from the alternate path by an isolating region as in AODV-DM (see figure 3). Once multiple paths are constructed and traffic begins to flow through the primary path, the source station (STA) monitors the connection for congestion. Congestion detection is done by TCP Vegas by monitoring the round trip time (RTT). Once congestion is detected the source STA initiates alternate path switch by transmitting a rate-based congestion detection (RCDN) message to the mesh access point (MAP) to which it is associated. The MAP then diverts data to the alternate path and informs the source STA through a path-alternation notification (PAN) If the primary path fails or if congestion is detected, it is the source that performs path switching. Therefore local recovery from path failure is not possible in PATR. Through simulations in NS2 the authors show that TCP connections are more stable and robust with part.

\subsection{MRDWMN}

Long Le [13] present a multipath routing design for WMNs, for optimising throughput, taking into account, interference and channel load of links. The design consists of five components: topology discovery, resource discovery, multipath computation, path setup and maintenance and multipath forwarding. OLSR is used for topology discovery and it allows the protocol to create a network graph. In resource discovery phase, periodic broadcast of HELLO messages allows nodes to deduce the channel load of their one-hop neighbours. Nodes then piggyback this information on OLSR's Topology Control messages which allows a node to derive estimations of available bandwidth at other nodes. Using this information the multipath computation algorithm seeks to find less frequently used paths that can provide a minimum bandwidth guarantee. For every source, the algorithm starts by finding the neighbour that has the lowest visit count, which can provide the minimum bandwidth required. If such a node is found the node is added to the path and its visit counter is increment and the local bandwidth estimation of the node is reduced by the minimum bandwidth. The reduction of bandwidth allows traffic to be routed through nodes that can provide the minimum bandwidth required. Also the algorithm increments the visit counter to ensure that less frequented nodes are chosen. The found node is then processed similarly until the destination is reached at which point the path is added to the path set. A reverse path is also maintained by keeping track of the parent of each chosen node along the path. Once multiple paths are selected a path setup message is sent along each path. An intermediate node on receiving the message inserts a new entry for this path in its path table and forwards it to the next node. In this manner the path setup message propagates along the path and arrives at the destination which then sends a path confirm packet back to the source. This completes the path setup process and data can now be transmitted along this path. Path setup messages additionally assist in congestion signalling. Two fields in the path setup message contain information on the amount of data the source intends to send which informs intermediate nodes of the source's bandwidth requirements and about the available bandwidth at the bottleneck node along the path. Initially this second field is initialised to infinity and each node along the path decrements the value if it detects itself to be the bottleneck. The final information about the bottleneck node is sent back to the source by the destination via the previously mentioned path confirm packet. The source uses this information to calculate the quantum of data traffic to be allotted to each of the multiple paths. The negative effects on TCP performance is countered by not splitting burst data into different paths. This allows the packets of the burst data to arrive in sequence at the receiver. If however data packets do arrive out of order, a packet buffer maintained at the destination for a certain interval, which allows data to be reordered before it is handed to TCP. The design does not allow for local recovery. If a path fails, it is the source that has to decide which alternate path should be used next. As with other protocols suffering from the problem, it adds latency to the process of recovery after a path failure. Also, the design does not provide a mechanism to deal with the route coupling problem.

Comparison of the design with OLSR in the NS-2 simulator shows that throughput is greatly increased. The authors also make a comparison between OLSR and MRDWMN with HTTP traffic and analyse the response times. Here too, MRDWMN outperforms OLSR.

\subsection{SDMR}

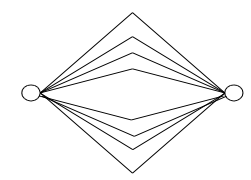

(a) Spatial separation of paths
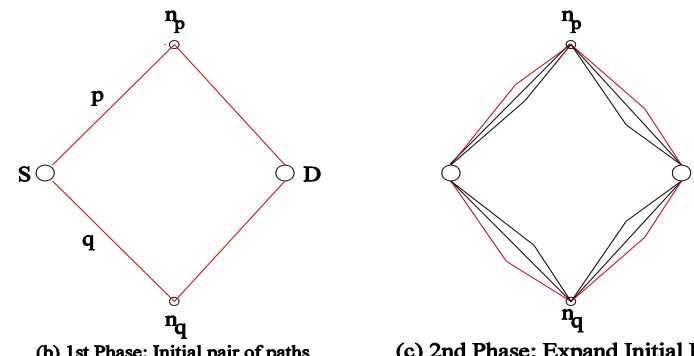

(c) 2nd Phase: Expand Initial Pair

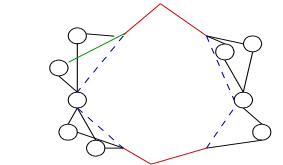

(d) 3rd Phase: Separate near endpoints

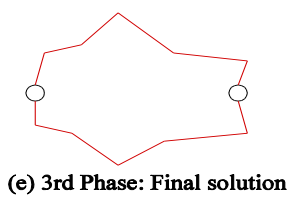

Fig. 7. Spatial Separation in SDMR using PCA [5] 
Table 1. Comparision of Multipath Routing Protocols for WMNs

\begin{tabular}{|c|c|c|c|c|c|c|c|}
\hline Protocol & Path Discovery & Path Maintenance & Traffic Distribution & TCPPerformance & $\begin{array}{l}\text { Distinguishing } \\
\text { Feature(s) }\end{array}$ & Protocol Performance & Shortcomings \\
\hline MMESH & $\begin{array}{l}\text { Proactive gateway } \\
\text { advertisement } \\
\text { messages }\end{array}$ & $\begin{array}{l}\text { MR periodically monitor paths } \\
\text { and are authorised to route } \\
\text { data along alternate paths in } \\
\text { case of route failures }\end{array}$ & $\begin{array}{l}\text { Round robin or based on } \\
\text { next hops congestion load } \\
\text { value }\end{array}$ & $\begin{array}{l}\text { Does not deal with TCP } \\
\text { performance }\end{array}$ & $\begin{array}{l}\text { Proactive messages are } \\
\text { sent by gateways are used } \\
\text { to setup multiple paths }\end{array}$ & $\begin{array}{l}\text { Simulations show better } \\
\text { throughput of flows in } \\
\text { comparison to AODV }\end{array}$ & $\begin{array}{l}\text { Route-Coupling } \\
\text { Cannot use multi-radio } \\
\text { Cannot use multiple- } \\
\text { gateways }\end{array}$ \\
\hline ASMRP & Similar to MMESH & Similar to MMESH & $\begin{array}{l}\text { Based on congestion level } \\
\text { of next hop }\end{array}$ & $\begin{array}{l}\text { Does not deal with TCP } \\
\text { performance }\end{array}$ & $\begin{array}{l}\text { Similar to MMESH but } \\
\text { adapted for multi-radio } \\
\text { multi-channel mesh }\end{array}$ & $\begin{array}{l}\text { Through simulations it is } \\
\text { shown better throughput, } \\
\text { delay \& packet delivery } \\
\text { than AODV, } \\
\text { MMESH,MMR,CAM- } \\
\text { ASMRP }\end{array}$ & $\begin{array}{l}\text { Dependent of channel } \\
\text { assignment scheme } \\
\begin{array}{l}\text { Doesn't support multiple } \\
\text { gateways }\end{array}\end{array}$ \\
\hline 2CPR & Not specified & Not specified & $\begin{array}{l}\text { Concurrent use of pair of } \\
\text { paths }\end{array}$ & $\begin{array}{l}\text { Does not deal with TCP } \\
\text { performance }\end{array}$ & $\begin{array}{l}\text { A pair of paths are } \\
\text { computed from a source, } \\
\text { based on a metric to two } \\
\text { gateways }\end{array}$ & $\begin{array}{l}\text { Simulations shows } \\
\text { increase in the probability } \\
\text { of finding routes. } \\
\text { Significantly increases } \\
\text { network throughput } \\
\text { compared to } 2 \text { SPR \& } \\
\text { AODV-DM }\end{array}$ & $\begin{array}{l}\text { Does not allow local } \\
\text { recovery from route } \\
\text { failure }\end{array}$ \\
\hline AODV-DM & $\begin{array}{l}\text { Two round path } \\
\text { discovery using } \\
\text { RREQs messages }\end{array}$ & Not specified & $\begin{array}{l}\text { Concurrent use of primary } \\
\text { and secondary paths }\end{array}$ & $\begin{array}{l}\text { Suggests use of path } \\
\text { aware TCP such as } \\
\text { SCTP }\end{array}$ & $\begin{array}{l}\text { Primary and secondary } \\
\text { paths separated by an } \\
\text { insulating region of nodes }\end{array}$ & $\begin{array}{l}\text { Simulations scenarios: } \\
\text { UDP: Increases success } \\
\text { rate. Decreases end to end } \\
\text { delay } \\
\text { TCP: Decreases } \\
\text { congestion window } \\
\text { oscillation Increases } \\
\text { goodput }\end{array}$ & $\begin{array}{l}\text { Two-round route } \\
\text { discovery adds latency } \\
\text { Nodes in Insulating region } \\
\text { cannot route data } \\
\text { No local recovery from } \\
\text { link failures }\end{array}$ \\
\hline MHRP & $\begin{array}{l}\text { Three stages of routes } \\
\text { discovery: route } \\
\text { request, route } \\
\text { formation and route } \\
\text { reply }\end{array}$ & $\begin{array}{l}\text { Each node has all possible } \\
\text { paths and switches to alternate } \\
\text { route when a link is lost }\end{array}$ & Not specified & $\begin{array}{l}\text { Does not deal with TCP } \\
\text { performance }\end{array}$ & $\begin{array}{l}\text { Hybrid routing that has } \\
\text { four component protocols } \\
\text { for different parts of } \\
\text { WMN. }\end{array}$ & $\begin{array}{l}\text { Simulation shows Better } \\
\text { throughput vis-sa-vis other } \\
\text { protocols. } \\
\text { Provides increased } \\
\text { security with less } \\
\text { overhead }\end{array}$ & $\begin{array}{l}\text { Does not address route } \\
\text { coupling problem }\end{array}$ \\
\hline DIPRO & Not specified & Not specified & $\begin{array}{l}\text { Multiple primary paths } \\
\text { can be used concurrently }\end{array}$ & $\begin{array}{l}\text { Does not deal with TCP } \\
\text { performance }\end{array}$ & $\begin{array}{l}\text { Uses interference between } \\
\text { link to provide protection } \\
\text { for paths. }\end{array}$ & $\begin{array}{l}\text { Simulations shows } \\
\text { increases in the satisfied } \\
\text { ratio and decreases in the } \\
\text { running time in } \\
\text { comparison to DPR and } \\
\text { AODV }\end{array}$ & $\begin{array}{l}\text { Not be suitable for sparse } \\
\text { networks }\end{array}$ \\
\hline PATR & $\begin{array}{l}\text { Similar to } \\
\text { AODV_DM }\end{array}$ & $\begin{array}{l}\text { On detection of congestion the } \\
\text { intermediate MR switches to } \\
\text { alternate path }\end{array}$ & $\begin{array}{l}\text { Alternate path is used as } \\
\text { backup of primary path }\end{array}$ & $\begin{array}{l}\text { Uses Vegas TCP which } \\
\text { initiates BaseRTT on } \\
\text { receipt of path } \\
\text { alternation notification }\end{array}$ & $\begin{array}{l}\text { Offers fast switching to } \\
\text { alternate path in case of } \\
\text { failure of primary path }\end{array}$ & $\begin{array}{l}\text { Simulations show increase } \\
\text { stability in TCP } \\
\text { performance }\end{array}$ & $\begin{array}{l}\text { No local recovery from } \\
\text { path failures }\end{array}$ \\
\hline MRDWMN & $\begin{array}{l}\text { Creates network graph } \\
\text { from OLSR topology } \\
\text { information }\end{array}$ & $\begin{array}{l}\text { Source periodically sends path } \\
\text { setup messages along } \\
\text { established paths }\end{array}$ & $\begin{array}{l}\text { Source performs traffic } \\
\text { shaping and splits traffic } \\
\text { amongst multiple paths } \\
\text { based on congestion } \\
\text { feedback from } \\
\text { intermediate nodes }\end{array}$ & $\begin{array}{l}\text { Two mechanisms: } 1 \text {. } \\
\text { Does not split burst data } \\
\text { into multiple paths and } \\
\text { 2. Packet buffer at the } \\
\text { receiver for out of order } \\
\text { packets }\end{array}$ & $\begin{array}{l}\text { Incorporates channel load } \\
\text { and interference while } \\
\text { computing paths }\end{array}$ & $\begin{array}{l}\text { Simulation shows better } \\
\text { throughput in comparison } \\
\text { to OLSR. Provides better } \\
\text { response times to HTTP } \\
\text { traffic }\end{array}$ & $\begin{array}{l}\text { No local recovery from } \\
\text { path failures } \\
\begin{array}{l}\text { Does not address route- } \\
\text { coupling problem }\end{array}\end{array}$ \\
\hline SDMR & $\begin{array}{l}\text { Uses OLSR like } \\
\text { mechanism that sends } \\
\text { link state information } \\
\text { only to to source on } \\
\text { demand }\end{array}$ & $\begin{array}{l}\text { Nodes monitor links and } \\
\text { inform source of link } \\
\text { breakages }\end{array}$ & $\begin{array}{l}\text { Concurrent use of pair of } \\
\text { paths }\end{array}$ & $\begin{array}{l}\text { Does not deal with TCP } \\
\text { performance }\end{array}$ & $\begin{array}{l}\text { Spatially disjoint paths are } \\
\text { computed following a } \\
\text { heuristic approach }\end{array}$ & $\begin{array}{l}\text { Simulations show fewer } \\
\text { control overhead in } \\
\text { comparison to AODV \& } \\
\text { AODVM. SDMR has } \\
\text { similar PDR to AODV. } \\
\text { But provides large spatial } \\
\text { separation between paths }\end{array}$ & $\begin{array}{l}\text { Not suitable for sparse } \\
\text { WMNS } \\
\text { Degree of spatial } \\
\text { separation near source and } \\
\text { destination low } \\
\text { No local recovery from } \\
\text { route failures }\end{array}$ \\
\hline
\end{tabular}

Spatially Disjoint Multipath Routing (SDMR) [5] computes spatially disjoint paths without the help of location information. The route coupling phenomena is minimized and the network is resilient against regional node failures where nodes of an entire region fail at the same time. Following an OLSR like approach, the connectivity graph is constructed by the source, on-demand, using connectivity information obtained from the set of multi-point relays (MPRs) and the 
destination. The information is acquired by flooding Topology Request (TREQs) messages. MPRss reply by sending their 1hop neighbour set in a Topology Reply (TREP) message. The 1-hop neighbour set is sent only if the set has changed since the last request from source $\mathrm{S}$. Once the source receives TREPs for all destinations it builds a topology graph to feed to the path calculation algorithm (PCA) which computes a pair of spatially disjoint paths. As the number of nodes in a network grows the computation of all possible paths between two nodes grows exponentially. PCA employs a heuristic approach that reduces the number of candidate paths from a source to a destination and from this reduced set derives a pair of spatially disjoint paths. To compute the reduced set of paths, PCA identifies generating nodes such that these nodes are equidistant in terms of number of hops to the source and destination (figure 7 (a)). The resultant shortest paths connecting the generating nodes to the source and destination will be small in number. A brute force approach is then applied to find a pair of disjoint paths (figure 7 (b)). The algorithm then proceeds to improve the solution by finding paths that are close to this pair as there can be multiple shortest paths between two nodes. It generates all shortest paths connecting the generating nodes to the source and destination and chooses the best pair of paths (figure 7 (c)). To increase spatial separation at the two endpoints of the path, the algorithm keeps the inner part of the path and connects the source and the destination to their previously unused two-hop neighbours (figure 7 (d)). This gives a new set of paths from which the pair that is most disjoint is arrived at (figure 7 (e)). By routing data through spatially separated pair of paths, SDMR, outperforms OLSR, AODV and AOMDV. However, in low density WMNs it may not find spatially disjoint paths in WMNs. Also, near the source and destination the degree of spatial separation achieved may be very low. Another limitation is that it does not allow for local recovery. If a link fails, the source nodes are informed via route error messages (RERR) so that they may direct traffic through alternate paths increasing the delay in delivering traffic. The protocol does not deal with out of order arrival of packets which degrades TCP performance. Compared to AODV and AODVM, SDMR generates much fewer number of RREQ and RREP messages. SDMR has similar packet deliver ratio as AODV in the face of node mobility. In fact in SDMR packet delivery rates drops at a faster than in AODV when there is node mobility. However, SDMR provides a large degree of spatial separation between multiple paths.

\section{DISCUSSION}

Multipath routing offers considerable gains over single path routing but at the cost of increased complexity and routing overhead. As packets are distributed between multiple paths, they may arrive at the destination out of sequence. Unless special measures are adopted to reassemble the out of order packets, it can have a detrimental effect on TCP performance. But we show that most multipath routing algorithms ignore this aspect $[17,16,3,21,5]$. A good multipath routing protocol must therefore take into consideration the effect it has on TCP and also deal with route-coupling, provide load balancing, and fault tolerance with low control overhead. Some schemes aim to solve the route-coupling problem by creating a protection region of nodes around the primary path as in [8] while others use the very paths interfering with the primary path to provide protection to it [10]. These strategies may, however lead to increased latency in the route discovery process and may also not be suitable for sparse WMNs where depleted node density does not afford nodes to be used as guards for the primary path. Other protocols that aim to discover node disjoint or spatially node disjoint paths [5] may also not be suitable for sparse WMNs for the same reason. Multipath routing protocols must also exploit the unique characteristic of WMNs, like use of multi-radio [16] which mitigates the effects of interference and also have the ability to split traffic to multiple gateways. One of the benefits of multipath routing is its ability to recover from route failures quickly. However protocols must explicitly ensure that such recovery is possible. $[17,16,21,4]$ allow intermediate nodes to switch transmission to an alternate path on detection of route failures, while $[13,5]$ inform the source node of failures, which then has to choose an alternate route, which slows the process of recovery. It has been shown that none of the protocols provide a holistic solution taking into account all these factors. Given that there are a large number of trade-offs involved, designing a routing protocol that comprehensively addresses all issues is a challenging task and remains and open research issue. Table 1 shows a comparison matrix that provides an overview of the protocols and can help in identifying the strength and weaknesses of each.

\section{CONCLUSION}

In this survey a representative set of multipath routing algorithms for wireless mesh networks have been analysed. It has been shown that all protocols do not address all the issues of multipath routing and a unifying solution remains elusive. This study can serve as a ready reference for the protocols analysed and be a starting point for new researchers in the area of multipath routing design for WMNs. As the field of multipath routing in wireless mesh networks is ever changing and new protocols are invented, future work could extend the study to include the advances in the area.

\section{REFERENCES}

[1] S. Adibi and S. Erfani. A multipath routing survey for mobile ad-hoc networks. CCNC 2006. 2006 3rd IEEE Consumer Communications and Networking Conference, 2006., 2:984-988, 2006.

[2] I.F. Akyildiz, X. Wang, and W. Wang. Wireless Mesh Networks: A Survey. Computer Networks, 47(4):445487, 2005.

[3] Chia-hung Chang and Wanjinn Liao. On Multipath Routing in Wireless Mesh Networks with Multiple Gateways. In IEEE GlobeCom, pages 1-5, 2010.

[4] Yi-Ting Chien, Mong-Fong Horng, Yu-Cheng Sheu, JengPong Hsu, Yau-Hwang Kuo, and Chin-Shiun Shieh. An Alternative-Path Approach to Improve Robustness of TCP Connections in Wireless Mesh Networks. 2010 Sixth International Conference on Intelligent Information Hiding and Multimedia Signal Processing, pages 248251, October 2010.

[5] Juan J. Galvez, Pedro M. Ruiz, and Antonio F.G. Skarmeta. Multipath routing with spatial separation in wireless multihop networks without location information. Computer Networks, 55(3):583-599, February 2011.

[6] Y Ganjali and A Keshavarzian. Load balancing in ad hoc networks: single-path routing vs. multi-path routing. INFOCOM 2004. Twenty-third AnnualJoint Conference of the IEEE Computer and Communications Societies, pages $0-5,2004$.

[7] M. Gerla. TCP performance over multipath routing in mobile ad hoc networks. IEEE International Conference on Communications, 2003. ICC '03., 2:1064-1068, 2003. 
[8] Xuhui Hu and Myung J. Lee. An efficient multipath structure for concurrent data transport in wireless mesh networks. Computer Communications, 30(17):33583367, November 2007.

[9] Yun Hu, Weiqing He, Shoubao Yang, and Yuan Zhou. Multigateway multi-path routing protocol for $802.11 \mathrm{~s}$ WMN. 2010 IEEE 6th International Conference on Wireless and Mobile Computing, Networking and Communications (WiMob), pages 308-315, 2010.

[10] Farah Kandah, Weiyi Zhang, Chonggang Wang, and Juan Li. Diverse Path Routing with Interference and Reusability Consideration in Wireless Mesh Networks. Mobile Networks and Applications, 17(1):100-109, March 2011.

[11] S.V. Krishnamurthy and S.K. Tripathi. Effects of multipath routing on TCP performance in ad hoc networks. IEEE Global Telecommunications Conference, 2004. GLOBECOM '04., 6:4125-4131, 2004.

[12] R. Krishnan and J.a. Silvester. Choice of allocation granularity in multipath source routing schemes. INFOCOM'93. Proceedings. Twelfth Annual Joint Conference of the IEEE Computer and Communications Societies. Networking: Foundation for the Future, IEEE, pages 322-329, 1993.

[13] Long Le. Multipath Routing Design for Wireless Mesh Networks. 2011 IEEE Global Telecommunications Conference GLOBECOM 2011, pages 1-6, December 2011.

[14] SJ Lee and M Gerla. Split multipath routing with maximally disjoint paths in ad hoc networks. IEEE International Conference on Communications, 2001. ICC 2001., 2001.

[15] S Mueller, RP Tsang, and D Ghosal. Multipath routing in mobile ad hoc networks: Issues and challenges. Performance Tools and Applications to Networked Systems, 2004.

[16] Deepti S. Nandiraju, Nagesh S. Nandiraju, and Dharma P. Agrawal. Adaptive state-based multi-radio multichannel multi-path routing in Wireless Mesh Networks.
Pervasive and Mobile Computing, 5(1):93-109, February 2009.

[17] D.S Nandiraju, N.S Nandiraju, and D.P. Agrawal. Multipath routing in wireless mesh networks. 2006 IEEE international conference on Mobile adhoc and sensor systems (MASS), 45221:41-46, 2006.

[18] L Ong. An introduction to the stream control transmission protocol (SCTP). Technical report, 2002.

[19] M.R. Pearlman, Z.J. Haas, P. Sholander, and S.S. Tabrizi. On the impact of alternate path routing for load balancing in mobile ad hoc networks. 2000 First Annual Workshop on Mobile and Ad Hoc Networking and Computing. MobiHOC (Cat. No.OOEX444), pages 3-10, 2000.

[20] D. Saha, S. Roy, S. Bandyopadhyay, T. Ueda, and S. Tanaka. An adaptive framework for multipath routing via maximally zone-disjoint shortest paths in ad hoc wireless networks with directional antenna. GLOBECOM '03. IEEE Global Telecommunications Conference (IEEE Cat. No.03CH37489), 1:226-230, 2003.

[21] Muhammad Shoaib Siddiqui, Syed Obaid Amin, Jin Ho Kim, and Choong Seon Hong. MHRP: A Secure MultiPath Hybrid Routing Protocol for Wireless Mesh Network. MILCOM 2007 - IEEE Military Communications Conference, pages 1-7, October 2007.

[22] Mohammed Tarique, Kemal E. Tepe, Sasan Adibi, and Shervin Erfani. Survey of multipath routing protocols for mobile ad hoc networks. Journal of Network and Computer Applications, 32(6):1125-1143, November 2009.

[23] Jack Tsai and Tim Moors. A review of multipath routing protocols: From wireless ad hoc to mesh networks. In ACoRN early career researcher workshop on wireless multihop networking, 2006.

[24] Zhenqiang Ye, Srikanth V Krishnamurthy, and Satish K Tripathi. A framework for reliable routing in mobile ad hoc networks. In INFOCOM 2003. Twenty-Second Annual Joint Conference of the IEEE Computer and Communications. IEEE Societies, volume 01, pages 270 280

IEEE, 